\title{
Age-Related Insulin-Like Growth Factor Binding Protein-4 Overexpression Inhibits Osteogenic Differentiation of Rat Mesenchymal Stem Cells
}

\author{
Jinhui Wu Chao Wang Xiong Miao Yungang Wu Jiabin Yuan Muchen Ding \\ Jingfeng Li Zhicai Shi
}

Orthopedic Department of Changhai Hospital, Shanghai, China

\section{Key Words}

Aging - Insulin-like growth factor binding protein-4 - Bone marrow-derived mesenchymal stem cells $•$ Osteogenic differentiation

\begin{abstract}
Background/Aims: Insulin-like growth factor binding proteins (IGFBP) play important roles in bone metabolism. IGFBP4 is involved in senescent-associated phenomena in mesenchymal stem cells (MSCs). The goal of the present study was to determine whether age-related IGFBP4 overexpression is associated with the impaired osteogenic differentiation potential of aged bone marrow derived MSCs. Methods: MSCs were isolated from Sprague-Dawley rats aged 3-26 months. The bone morphogenetic protein (BMP)-2-induced osteogenic differentiation of rat MSCs was assessed by analyzing the expression levels of osteoblast marker genes [runtrelated transcription factor 2 (RUNX2), alkaline phosphatase (ALP), and osteocalcin (OC)], ALP activity and calcification. Results: Our study showed that IGFBP4 mRNA and protein expression increased with age in parallel with impaired osteogenic differentiation of MSCs cultured in BMP2-containing osteogenic medium, as evidenced by the downregulation of osteoblast marker genes, and decreased ALP activity and calcium deposits. IGFBP4 overexpression impaired BMP2-induced osteogenic differentiation potential of young MSCs, whereas IGFBP4 knockdown restored the osteogenic potency of aged MSCs. Moreover, IGFBP4 knockdown stimulated the activation of Erk and Smad by increasing phosphorylation. Conclusion: Collectively, our results demonstrate that IGFBP4 overexpression plays a role in the impairment of MSC differentiation potential via the Erk and Smad pathways, suggesting potential targets to improve MSC function for cell therapy applications.
\end{abstract}




\section{Introduction}

Bone marrow-derived mesenchymal stem cells (BM-MSCs) are self-renewing multipotent cells with the potential to differentiate into several lineages, such as osteogenic, adipogenic, chondrogenic, and neurogenic lineages [1,2]. Because of their multipotent differentiation potential, MSCs are important tools for tissue regeneration and have therefore been researched extensively [3]. While an increasing number of studies have demonstrated that MSCs are an excellent choice for cell-based tissue engineering therapy for bone [4-6], the osteogenic capacity of MSCs appears to decline with age or passages of cultivated MSCs $[7,8]$. However, the mechanisms associated with age-related changes in MSC osteogenic potential remain unclear.

The insulin-like growth factor (IGF) system, including IGFs-I, and -II and IGF binding proteins (IGFBPs), plays an important role in many cellular processes, including cell growth, differentiation, and cellular senescence [9]. The IGFBP family comprises six structurally related proteins (IGFBP-1 to -6) characterized by cell- and tissue-dependent expression patterns that bind to IGF with high affinity, restricting binding of IGF to the IGF receptor, resulting in the inhibition of cell proliferation, differentiation, and survival [10,11]. IGFBP4 is a potent inhibitor of IGF-I action. Pregnancy-associated plasma protein-A (PAPP-A) can cleave IGFBP4 and liberate active IGF-I [12]. A previous study showed that reduced PAPP-A expression is associated with the upregulation of IGFBP4 expression and downregulation of IGF-IR level, which may be involved in low invasive potential and growth rate in antisense (AS)-PAPP-A cells in response to IGF-I [13]. IGFBP5 or a recombinant adenovirus expressing wild-type IGFBP5 prevented BMP2-induced osteogenic differentiation, whereas an analog with reduced IGF binding capacity was ineffective, suggesting that IGFBP5 interferes with the function of IGF [14]. Similarly, IGFBP3 overexpression was shown to inhibit the osteogenic differentiation of adipose derived stem cells. Serum levels of IGFBP4 showed negative correlation with calcaneal bone mineral density among post-menopausal SouthIndian women [15]. Importantly, expression of IGFBP4 inhibits BMP-9-induced ALP activity and matrix mineralization in MSCs [16], suggesting that IGFBP4 is involved in the osteogenic potency of MSCs.

Aging may decrease the osteogenic potency of MSCs [17,18]. IGFBP4 levels increase with aging and menopause [15]. IGFBP4 and IGFBP7 were identified as factors secreted from senescent MSCs and shown to induce premature senescence and apoptosis when added to young MSCs, suggesting that they play a role in the processes of aging in MSCs [19]. On the basis of these observations, we proposed the hypothesis that IGFBP4 expression in MSCs may increase with aging, and this age-related IGFBP4-overexpression inhibits the osteogenic differentiation potential of MSCs.

In the present study, we examined the effects of aging on the differentiation potential of MSCs and the role of IGFBP4 expression in cells isolated from rats of different ages and explored the underlying mechanisms.

\section{Materials and Methods}

\section{Isolation and culture of BM-MSCS}

All procedures were performed in accordance with an animal protocol approved by the Second Military Medical University Institutional Animal Care and Use Committee. BM-MSCs were isolated and harvested as previously described [20]. BM-MSCs were isolated from bone marrow samples of SpragueDawley rats aged 3 to 26 months. Samples were diluted with PBS, centrifuged at $400 \times$ for $30 \mathrm{~min}$, and the buffy coat containing mononuclear cells was plated in $\alpha$-MEM (Life technologies; Carlsbad, CA) containing $10 \%$ fetal bovine serum (FBS), $100 \mathrm{U} / \mathrm{ml}$ penicillin and $100 \mathrm{mg} / \mathrm{l}$ streptomycin (Life technologies) at $37^{\circ} \mathrm{C}$ in a humidified atmosphere with $5 \% \mathrm{CO}_{2}$. Non-adherent cells were discarded after $24 \mathrm{~h}$ and the medium was replaced, with fresh medium added every 4 days thereafter. Cells in passages $\leq 3$ were used in the following experiments.

\section{KARGER}




\section{Cellular Physiology Cell Physiol Biochem 2017;42:640-650 \begin{tabular}{l|l} 
DOI: 10.1159/000477873 & $\begin{array}{l}\text { O 2017 The Author(s). Published by S. Karger AG, Basel } \\
\text { www.karger.com/cpb }\end{array}$
\end{tabular} \\ Wu et al.: IGFBP4 Overexpression Inhibits Osteogenic Differentiation}

\section{Flow cytometry}

For flow cytometry detection of CD29, CD90, and CD34 expression, cells were washed in PBS and stained with the corresponding FITC-labeled antibodies or FITC-labeled IgG for $30 \mathrm{~min}$ at room temperature. Cells were analyzed using a FACSCalibur flow cytometer (BD Biosciences, San Jose, CA, USA).

\section{Senescence-associated $\beta$-galactosidase staining}

The $\beta$-Galactosidase ( $\beta$-gal) assay was performed by using the SA- $\beta$-gal staining kit (Beyotime, China) according to the manufacturer's instructions. MSCs were washed with PBS at least 3 times and fixed for 15 min. After removing the fixative, cells were washed with PBS and then stained with $\beta$-gal solution at $37^{\circ} \mathrm{C}$ overnight.

\section{qRT-PCR analysis}

Total RNA was extracted using the TRIzol reagent and reverse transcribed using random primers and reverse transcriptase (Invitrogen, Carlsbad, CA). Real-time PCR reaction was performed in a Rotor-Gene 3000 System (Corbett Research, Morklake, Australia) using SYBR Green PCR kit (TaKaRa, Shiga, Japan). All quantitation was normalized to an endogenous control $\beta$-actin. The data were analyzed using the $2^{-\Delta \Delta \mathrm{CT}}$ method.

\section{Western blot analysis}

Total protein was extracted from MSCs using RIPA buffer (Beyotime, China) and protein concentration was determined using the BCA protein assay kit (Bio-Rad Laboratories, Hercules, CA) as previously described [21]. Proteins were separated by $10 \%$ SDS-PAGE and transferred to nitrocellulose membranes, which were incubated with primary antibodies against IGFBP4, Erk1/2, Smad1/5/8, p-Erk1/2, p-Smad1/5/8 or $\beta$-actin (Santa Cruz Biotechnology, Dallas, TX) overnight at $4^{\circ} \mathrm{C}$, followed by incubation in the corresponding HRPconjugated secondary antibodies (Santa Cruz Biotechnology) for $45 \mathrm{~min}$ at room temperature. Bands were visualized using an enhanced chemiluminescence (ECL) reagent (Beyotime).

\section{Lentivirus production and transduction}

The lentivral vectors encoding full length of rat IGFBP4 or short hairpin RNA (shRNA) against IGFBP4 were purchased from Hanbio (Shanghai, China). Control lentivral vectors were carrying no gene. MSCs were seeded at a density of $5 \times 10^{3}$ in 96 well plates and grown for $24 \mathrm{~h}$ before changing the medium to antibioticfree medium. Transducing lentiviral particles expressing IGFBP4 or IGFBP4 shRNA were added to reach a multiplicity of infection of 80 units and cells were cultured for $72 \mathrm{~h}$. The efficacy was determined by western blotting.

\section{Osteogenic differentiation}

For osteogenic differentiation, MSCs were cultured in growth medium ( $\alpha$-MEM supplemented with $10 \% \mathrm{FBS}$ and $1 \%$ penicillin and streptomycin) supplemented with $50 \mu \mathrm{g} / \mathrm{ml}$ ascorbic acid, $10 \mathrm{mM} \beta$-glycerol phosphate, and $200 \mathrm{ng} / \mathrm{ml} \mathrm{rh-BMP2}$. Differentiation into the osteoblast lineage was assessed by determining ALP activity by measuring p-nitrophenol in cell lysates. Alizarin red S staining was performed to detect matrix mineralization. In brief, cells were fixed with 70\% ice-cold ethanol, stained with an Alizarin Red Solution (2\%, pH 4.2, Sigma-Aldrich), and imaged using a microscope. The cells were solubilized using $10 \%$ ( $\mathrm{w} / \mathrm{v}$ ) cetylpyridinium chloride in $10 \mathrm{mM}$ sodium phosphate ( $\mathrm{pH} 7.0$ ), and absorbance was measured at 562 $\mathrm{nm}[22]$.

\section{Statistical analysis}

The results are shown as the mean \pm standard deviation (SD). Experiments were repeated three times independently using cells from different litters of mice or different passages. Comparison between control and treatment groups was done using one tailed Student's $t$-test. Values at $\mathrm{P}<0.05$ were considered significant. 


\section{Results}

IGFBP4 expression in rat MSCs increases with age

To determine the effect of aging on IGFBP4 expression, MSCs were isolated from 3-26 month-old rats. The identity of isolated cells was confirmed by flow cytometric analysis of surface markers, which showed that cells from both young (3-4 months old) and aged (23-26 months old) rats were positive for the mesenchymal markers CD29 and CD90 and negative for the hematopoietic lineage marker CD34 (Fig. 1A). Then, cellular senescence was assessed based on senescence-associated $\beta$-galactosidase (SA- $\beta$-gal) activity. As shown in Fig. 1B, the number of SA- $\beta$-gal-positive cells was significantly increased in MSCs derived from aged rats compared with those from young rats. qRT-PCR and western blot analysis of the expression of IGFBP4 showed that IGFBP4 was significantly upregulated with aging, with an approximately 4 -fold increase in mRNA expression and a 2.5-fold increase in protein levels in aged rats compared with young rats (Fig. 1C and D).

Age-related IGFBP4-overexpression is associated with inhibition of BMP2-induced osteogenic differentiation

To determine the association between age-related IGFBP4 overexpression and the osteogenic differentiation of MSCs, cells from young and aged rats were cultured in the presence of BMP2, and the expression of the osteogenic markers runt-related transcription factor 2 (Runx2, an essential transcription factor for osteoblast differentiation and bone formation), alkaline phosphatase (ALP, a marker of early osteogenic differentiation), and osteocalcin (OC, a critical marker of mature osteoblasts) was assessed by qRT-PCR. In young rats, BMP2 significantly upregulated the expression of Runx2, ALP, and OC by approximately 5-, 4- and 7-fold, respectively, after 10 days in culture. In aged rats, the upregulation of these osteoblast markers was significantly attenuated, with approximately 3-, 2-, and 4-fold upregulation of Runx2, ALP and OC, respectively, compared with the controls (Fig. 2A).

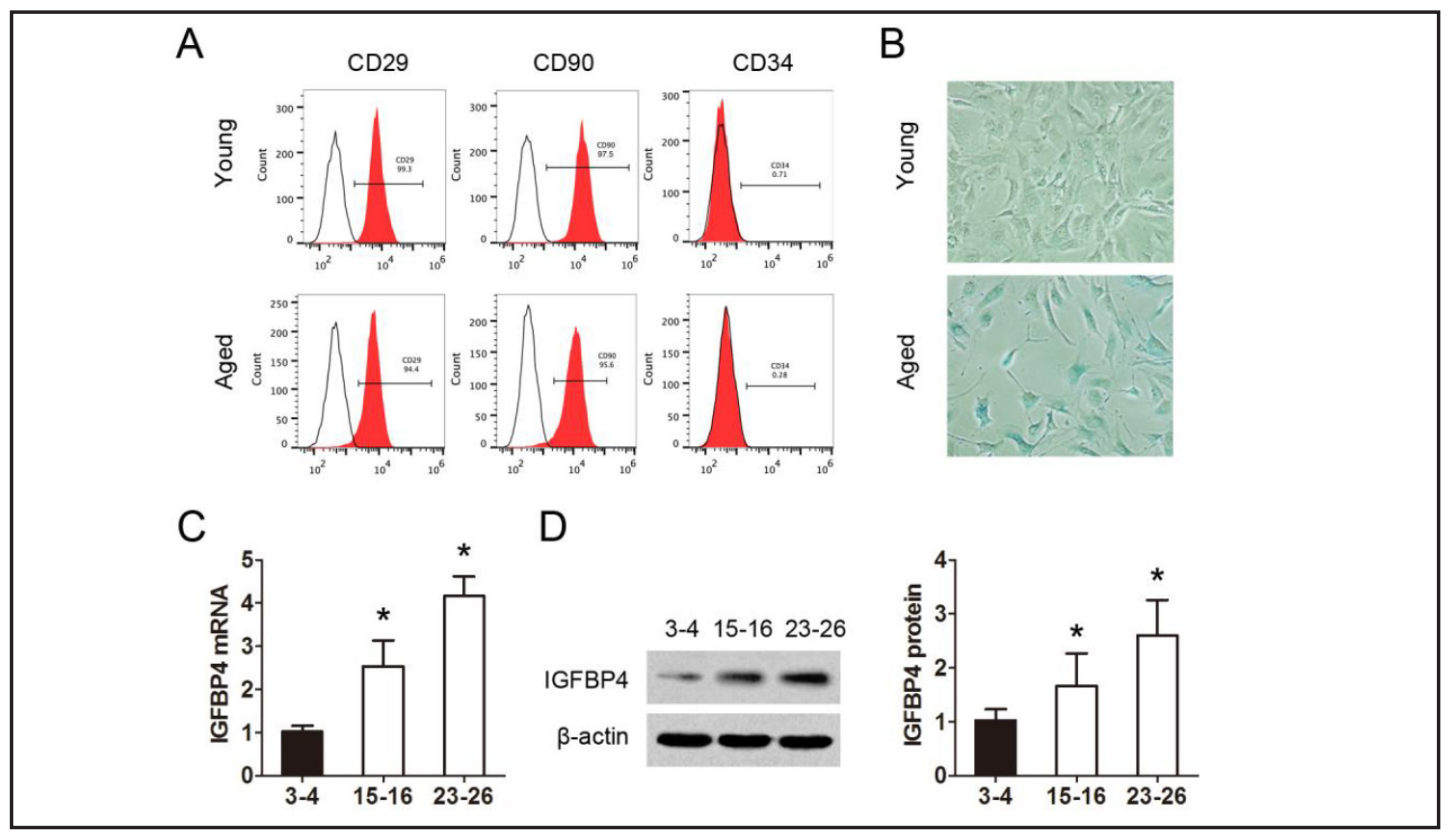

Fig. 1. IGFBP4 expression in rat MSCs increases with age. (A) Flow cytometric detection of CD29, CD90 and CD34 expression in MSCs derived from young (3-4 months old) and aged (23-26 months old) Sprague-Dawley rats. (B) SA- $\beta$-gal staining. (C) qRT-PCR analysis of IGFBP4 mRNA expression in MSCs derived from rats aged 3-26 months. (D) Western blot analysis of IGFBP4 protein expression and densitometric quantification of bands relative to the expression of $\beta$-actin. Data were expressed as the mean \pm SD from three independent experiments. ${ }^{*} P<0.05$ vs. MSCs of young rats at $3-4$ months old. 
A
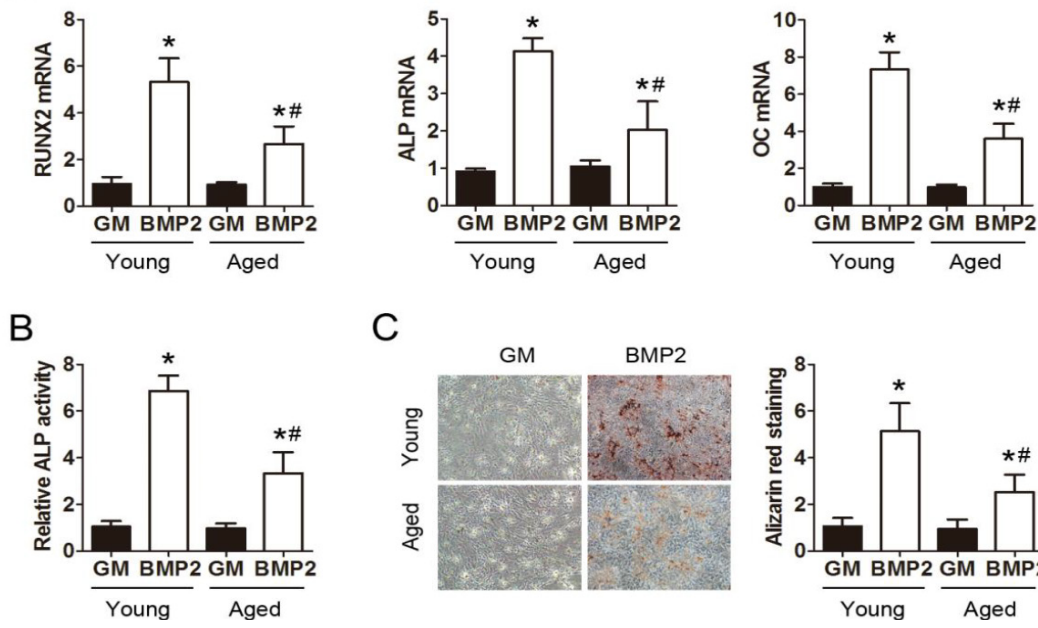

C
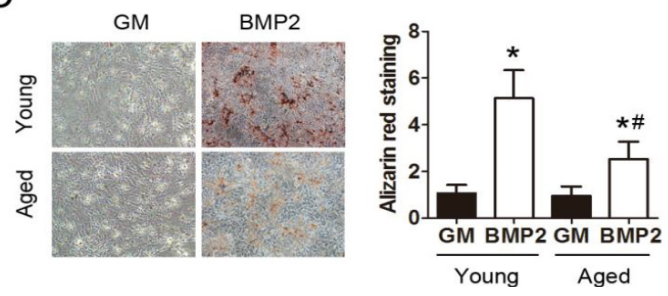

Fig. 2. Aging inhibits BMP2-induced osteogenic differentiation. (A) qRT-PCR analysis of the osteoblast markers RUNX2, ALP, and OC in MSCs cultured for 10 days in osteogenic medium (OM) containing BMP2 (BMP2) or growth medium (GM). (B) Alkaline phosphatase activity detection after 10 days of osteoblast differentiation. (C) Alizarin Red staining was performed on day 15. Data were expressed as the mean \pm SD from three independent experiments. ${ }^{*} P<0.05$ vs. GM. ${ }^{*} P<0.05$ vs. BMP2-stimulated young MSCs.

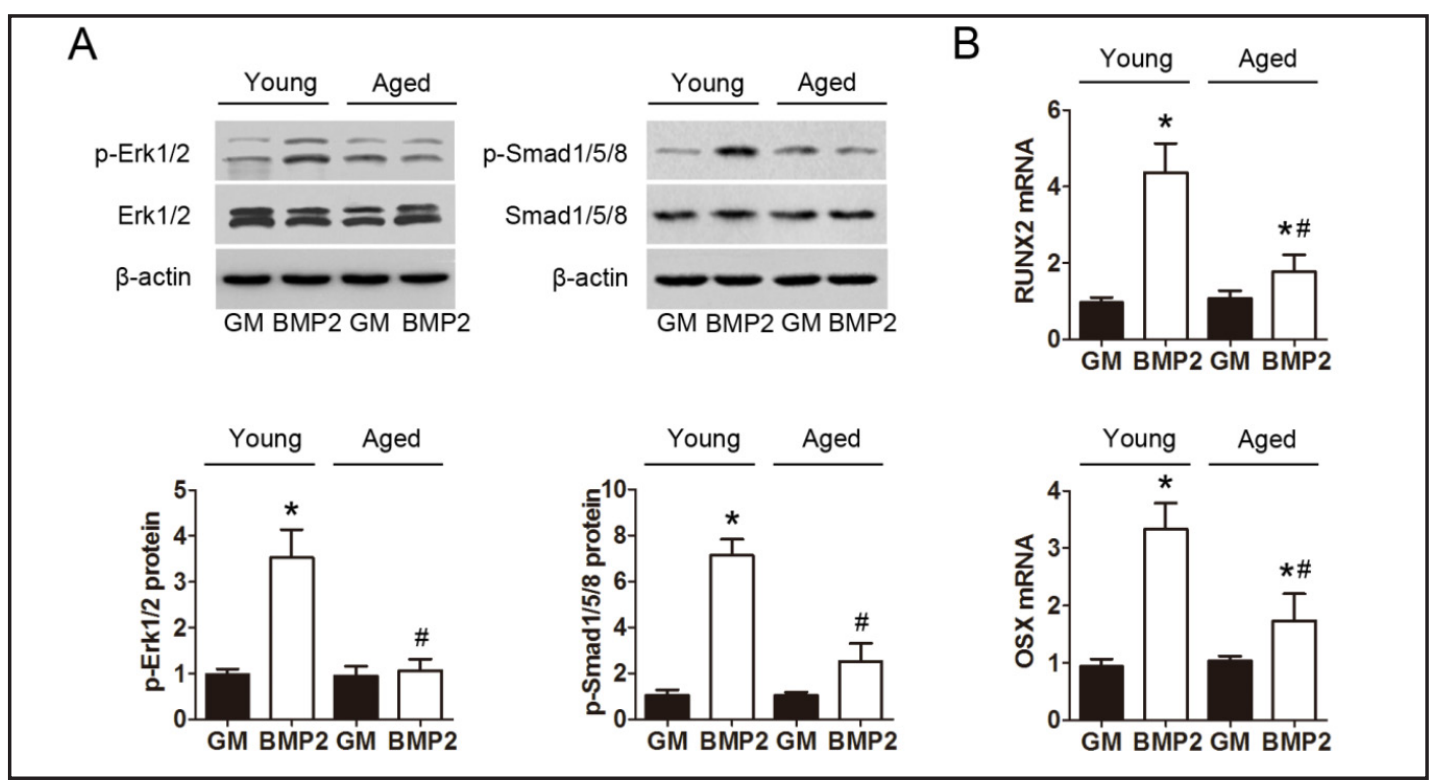

Fig. 3. Aging impairs BMP2-induced osteogenic differentiation by inhibiting the Erk and Smad-dependent pathways. (A) Western blot analysis of p-Erk1/2 and p- Smad1/5/8 relative to total Erk1/2 or Smad1/5/8 in young and aged MSCs incubated in OM containing BMP2 (BMP2) or growth medium (GM) for $60 \mathrm{~min}$. Graphs represent the results of densitometric quantification of bands relative to $\beta$-actin. (B) qRT-PCR analysis of downstream Runx2 and Osx expression in young and aged MSCs cultured in OM for $24 \mathrm{~h}$. Data represent the mean \pm SD from three independent experiments. ${ }^{*} P<0.05$ vs. GM. ${ }^{\#} P<0.05$ vs. BMP2-stimulated young MSCs.

Consistent with these results, the BMP2-induced increases in ALP activity and Alizarin red staining was significantly attenuated in aged rats compared with those in young rats (Fig. $2 \mathrm{~B}$ and $\mathrm{C}$ ). Taken together, these results indicated decreased osteogenic differentiation in 


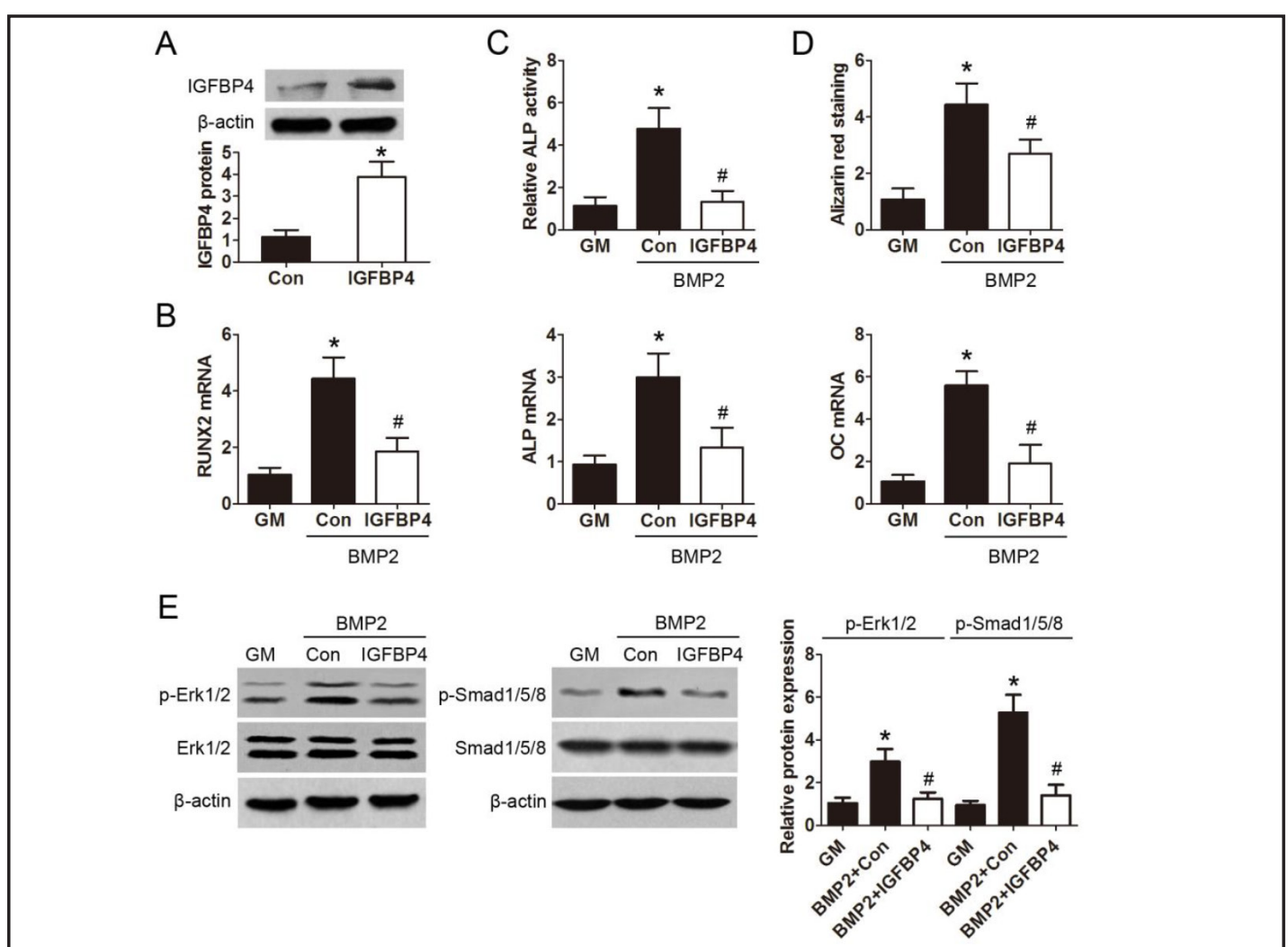

Fig. 4. Overexpression of IGFBP4 in young MSCs inhibits their osteogenic differentiation potential. (A) Young MSCs were infected with LV-IGFBP4 or LV-Con for $72 \mathrm{~h}$. IGFBP4 protein expression was detected by western blotting. Graphs represent the results of densitometric quantification of bands relative to $\beta$-actin. (B) qRT-PCR analysis of osteoblast marker genes (RUNX2, ALP, and OC) in IGFBP4-overexpressed cells after 10 days of culture in OM containing BMP2. (C) ALP activity in IGFBP4-overexpressed cells after 10 days of culture in OM containing BMP2. (D) Alizarin Red staining was performed on day 15. (E) Western blot analysis of p-Erk1/2 and p-Smad1/5/8 in IGFBP4-overexpressed cells incubated in OM containing BMP2 for 60 min. Graphs represent the results of densitometric quantification of bands relative to $\beta$-actin. Data represent the mean \pm SD from three independent experiments. ${ }^{*} P<0.05$ vs. GM. ${ }^{*} P<0.05$ vs. BMP2-stimulated MSCs control.

response to BMP2 stimulation in MSCs of aged rats that are known to express higher levels of IGFBP4 than that in MSCs of young rats.

Age-related impairment of BMP2-induced osteogenic differentiation is mediated by the inhibition of Erk and Smad-dependent pathways

Considering that extracellular signal-regulated kinase (Erk) and small mother against decapentaplegic (Smad) signaling pathways are responsible for transducing the BMP2 signal into the nucleus to regulate the transcription of osteogenesis-related genes [23-25], the activation of Erk1/2 and Smad1/5/8 in response to BMP2 was analyzed in MSCs from young and aged rats. Western blot analysis showed that BMP2 significantly upregulated the expression of p-Erk1/2 and p-Smad1/5/8 in MSCs from young rats, whereas this activation of Erk1/2 and Smad1/5/8 was suppressed in MSCs from aged rats (Fig. 3A). In parallel with the suppression of Erk and Smad signaling in aged rats, the transcription of Runx2 and Osx, the downstream target genes of the Erk and Smad pathways [26], was significantly decreased in aged rats (Fig. 3B). These results suggested that aging impairs the BMP2-induced osteogenic differentiation of MSCs by inhibiting Erk and Smad related pathways. 


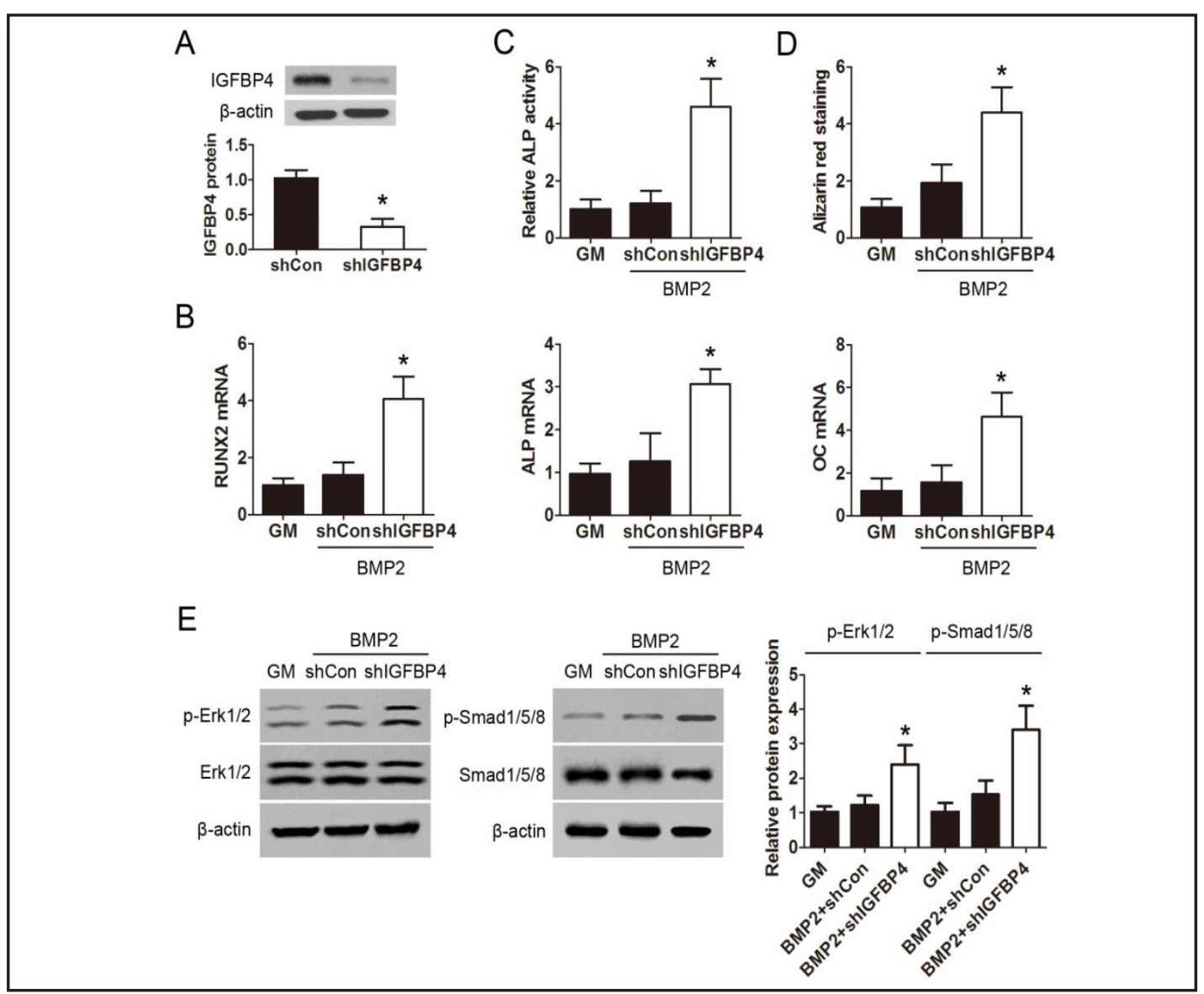

Fig. 5. Knockdown of IGFBP4 in aged MSCs restores their osteogenic differentiation potential. (A) Aged MSCs were infected with LV-shIGFBP4 or LV-shCon for $72 \mathrm{~h}$. IGFBP4 protein expression was detected by western blotting. Graphs represent the results of densitometric quantification of bands relative to $\beta$-actin. (B) qRT-PCR analysis of osteoblast marker genes (RUNX2, ALP, and OC) in IGFBP4-knockdown cells after 10 days of culture in OM containing BMP2. (C) ALP activity in IGFBP4-knockdown cells after 10 days of culture in OM containing BMP2. (D) Alizarin Red staining was performed on day 15. (E) Western blot analysis of p-Erk1/2 and p-Smad1/5/8 in IGFBP4-knockdown cells incubated in OM containing BMP2 for 60 min. Graphs represent the results of densitometric quantification of bands relative to $\beta$-actin. Data represent the mean \pm SD from three independent experiments. ${ }^{*} P<0.05$ vs. BMP2-stimulated MSCs control.

Lentivirus-mediated overexpression of IGFBP4 in young MSCs inhibits the osteogenic differentiation potential of MSCS

To verify the effect of IGFBP4 overexpression on the osteogenic differentiation potential of MSCs, we transfected young MSCs with lentivirus expressing IGFBP4. Western blot detection confirmed that transfection of IGFBP4 lentivirus significantly increased IGFBP4 protein level (Fig. 4A). After BMP2 stimulation, IGFBP4 overexpression markedly reduced the expression of osteoblast marker genes (RUNX2, ALP, and OC), ALP activity and mineralization compared with those in the control group (Fig. 4B-D). Then, Erk and Smad signaling pathways were analyzed in IGFBP4-overexpressed MSCs. The result showed that p-Erk1/2 and p-Smad1/5/8 expression was significantly decreased in IGFBP4-overexpressed MSCs compared with that in control cells (Fig. 4E). Collectively, these results suggest that IGFBP4 overexpression impairs the osteogenic differentiation potential of young MSCs via inhibiting Erk1/2 and Smad1/5/8 activation.

\section{KARGER}




\section{Cellular Physiology Cell Physiol Biochem 2017;42:640-650

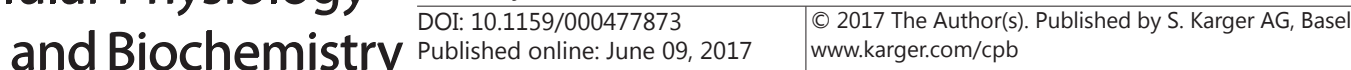 \\ Wu et al.: IGFBP4 Overexpression Inhibits Osteogenic Differentiation}

Knockdown of IGFBP4 in aged BMSCs restores the osteogenic differentiation potential of BMSCS

We further examined the effect of IGFBP4-deficiency on the osteogenic differentiation potential of aged MSCs. For this purpose, IGFBP4 was knocked down in MSCs from aged rats and osteogenic differentiation was assessed by measuring ALP expression and activity, and Runx2 and OC expression. Figure 5A shows the effective downregulation of IGFBP4 protein in IGFBP4 knockdown cells compared with control cells. The mRNA levels of Runx2, ALP, and OC, ALP activity and mineralization in BMP2-stimulated cells were significantly higher in IGFBP4 silenced cells than in control cells (Fig. 5B-D). Accordingly, p-Erk1/2 and p-Smad1/5/8 expression were markedly increased in IGFBP4 knockdown MSCs compared with control cells (Fig. 5E). Taken together, these results indicated that knockdown of IGFBP4 restored the osteogenic differentiation potential of aged MSCs via activation of Erk1/2 and Smad1/5/8.

\section{Discussion}

Aging and the age-related reduction in bone mass are associated with a decrease in the number of MSCs and the impairment of their osteogenic potential $[27,28]$. However, the mechanisms underlying the effect of aging on the differentiation potential and function of MSCs remain unclear. In the present study, we examined the factors and mechanisms involved in the age-related impairment of MSC differentiation into an osteoblastic lineage. We showed that IGFBP4 was upregulated in MSCs in correlation with aging, and overexpression and knockdown experiments showed that IGFBP4 plays an important regulatory role in MSC osteogenic differentiation possibly through the modulation of Erk and Smad dependent pathways.

IGFBP4 has been reported to play critical role in cell proliferation, differentiation, and senescence. For example, IGFBP4 promotes cardiomyocyte differentiation of embryonic stem cells [29]. In the cardiogenesis of mouse pluripotent stem cells, IGFBP4 significantly enhances the proliferation of cardiomyocytes [30]. Overexpression of GATA-4 significantly increases MSC differentiation into a myocardial phenotype, which was associated with the upregulation of IGFBP4 [31]. In the osteogenic differentiation of MSCs, IGFBP4 may compete for the binding of IGFs to their receptors, thus inhibiting IGF-II induced osteogenic potential [16]. IGFBP4 plays a key role in MSC senescence. Inhibition of IGFBP4 and -7 promoted cell growth and inhibited apoptosis, whereas addition of IGFBP4 and -7 induced MSC senescence and apoptosis in young MSCs [19]. IGFBP4 may have a pleiotropic effect on MSC biology. In the present study, we found that IGFBP4 expression increased with age in parallel with impaired osteogenic differentiation of MSCs cultured in BMP2-containing osteogenic medium, as evidenced by the downregulation of osteoblast marker genes (RUNX2, ALP, and OC), and decreased ALP activity and Alizarin red staining. IGFBP4 overexpression inhibited the BMP2-induced osteogenic differentiation potential of young MSCs, whereas knockdown of IGFBP4 restored the osteogenic differentiation potential of aged MSCs. Our study extends the understanding of the association between the upregulation of IGFBP4 and the inhibition of MSC osteogenic differentiation associated with aging.

To further understand the molecular mechanism associated with IGFBP4 upregulation and reduced osteogenic differentiation, we investigated the possibility that IGFBP4 may have an impact on signaling pathways that can crosstalk with BMP signaling. The phosphorylation of Erk and Smad, which have been shown to modulate bone formation and osteoblast differentiation, are usually triggered by BMPs [32,33]. A previous study showed that IGFBP5 overexpression suppresses melanoma cell growth and metastasis by reducing the phosphorylation of IGF1R, ERK1/2, and p38-MAPK kinases. Conversely, the phosphorylation of these kinases was enhanced in IGFBP5 knockdown cells [34]. IGFBP3 exerts its IGFindependent action by antagonizing BMP. Biochemical assays and in vitro experiments 


\section{Cellular Physiology Cell Physiol Biochem 2017;42:640-650 \begin{tabular}{l|l} 
DOI: 10.1159/000477873 & and Biochemistry \\
Published 2017 The Author(s). Published by S. Karger AG, Basel \\
www.karger.com/cpb
\end{tabular} \\ Wu et al.: IGFBP4 Overexpression Inhibits Osteogenic Differentiation}

revealed that IGFBP3 binds to BMP2 and inhibits BMP2-induced Smad signaling in cultured human cells [35]. Expression of the IGF antagonists IGFBP3 and IGFBP4 leads to inhibition of the IGF-2 effect on BMP-9-induced ALP activity and matrix mineralization. Mechanistically, IGF-2 is further shown to enhance the BMP-9-induced BMPR-Smad reporter activity and Smad1/5/8 nuclear translocation. PI3-kinase (PI3K) inhibitor LY294002 abolishes the IGF2 potentiation effect on BMP-9-mediated osteogenic signaling and can directly inhibit BMP-9 activity [16]. Consistent with these results, we observed that p-Erk1/2 and p-Smad1/5/8 levels were significantly decreased in IGFBP4-overexpressed MSCs and increased in IGFBP4 knockdown cells in the presence of BMP2. These results suggest that IGFBP4 modulates the BMP2 action on osteogenic differentiation through the Erk and Smad signaling pathways.

In conclusion, the present study showed that IGFBP4 plays a role in the impairment of MSC differentiation into an osteoblastic lineage associated with aging and this effect may be mediated by the inhibition of Erk and Smad dependent signaling. These results suggest that modulation of IGFBP4 expression is a potential strategy to improve the function of MSCs for use in cell therapy.

\section{Acknowledgments}

This work is supported by grants nos. 81672204 and 81372019 from the National Natural Science Foundation of China.

\section{Disclosure Statement}

The authors have declared that no conflict of interest exists.

\section{References}

1 Odabas S, Elcin AE, Elcin YM: Isolation and characterization of mesenchymal stem cells. Methods Mol Biol 2014;1109:47-63.

2 You L, Pan L, Chen L, Gu W, Chen J: Mir-27a is essential for the shift from osteogenic differentiation to adipogenic differentiation of mesenchymal stem cells in postmenopausal osteoporosis. Cell Physiol Biochem 2016;39:253-265.

3 Cui Y, Lu S, Tan H, Li J, Zhu M, Xu Y: Silencing of long non-coding rna nonhsat009968 ameliorates the staphylococcal protein a-inhibited osteogenic differentiation in human bone mesenchymal stem cells. Cell Physiol Biochem 2016;39:1347-1359.

-4 Kang IH, Jeong BC, Hur SW, Choi H, Choi SH, Ryu JH, Hwang YC, Koh JT: Microrna-302a stimulates osteoblastic differentiation by repressing coup-tfii expression. J Cell Physiol 2015;230:911-921.

-5 Izal I, Aranda P, Sanz-Ramos P, Ripalda P, Mora G, Granero-Molto F, Deplaine H, Gomez-Ribelles JL, Ferrer GG, Acosta V, Ochoa I, Garcia-Aznar JM, Andreu EJ, Monleon-Pradas M, Doblare M, Prosper F: Culture of human bone marrow-derived mesenchymal stem cells on of poly(l-lactic acid) scaffolds: Potential application for the tissue engineering of cartilage. Knee Surg Sports Traumatol Arthrosc 2013;21:17371750.

6 Zhang W, Chen J, Tao J, Jiang Y, Hu C, Huang L, Ji J, Ouyang HW: The use of type 1 collagen scaffold containing stromal cell-derived factor- 1 to create a matrix environment conducive to partial-thickness cartilage defects repair. Biomaterials 2013;34:713-723.

7 Joiner DM, Tayim RJ, Kadado A, Goldstein SA: Bone marrow stromal cells from aged male rats have delayed mineralization and reduced response to mechanical stimulation through nitric oxide and erk1/2 signaling during osteogenic differentiation. Biogerontology 2012;13:467-478.

8 Chung PL, Zhou S, Eslami B, Shen L, LeBoff MS, Glowacki J: Effect of age on regulation of human osteoclast differentiation. J Cell Biochem 2014;115:1412-1419. 


\section{Cellular Physiology Cell Physiol Biochem 2017;42:640-650

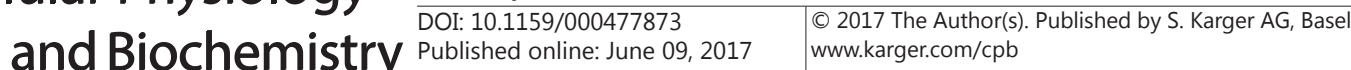

Wu et al.: IGFBP4 Overexpression Inhibits Osteogenic Differentiation

-9 Kim KS, Seu YB, Baek SH, Kim MJ, Kim KJ, Kim JH, Kim JR: Induction of cellular senescence by insulin-like growth factor binding protein-5 through a p53-dependent mechanism. Mol Biol Cell 2007;18:4543-4552.

10 Firth SM, Baxter RC: Cellular actions of the insulin-like growth factor binding proteins. Endocr Rev 2002;23:824-854.

11 Schneider MR, Wolf E, Hoeflich A, Lahm H: Igf-binding protein-5: Flexible player in the igf system and effector on its own. J Endocrinol 2002;172:423-440.

12 Lawrence JB, Oxvig C, Overgaard MT, Sottrup-Jensen L, Gleich GJ, Hays LG, Yates JR, 3rd, Conover CA: The insulin-like growth factor (igf)-dependent igf binding protein-4 protease secreted by human fibroblasts is pregnancy-associated plasma protein-a. Proc Natl Acad Sci U S A 1999;96:3149-3153.

13 Tanaka Y, Kobayashi H, Suzuki M, Hirashima Y, Kanayama N, Terao T: Genetic downregulation of pregnancyassociated plasma protein-a (papp-a) by bikunin reduces igf-i-dependent akt and erk1/2 activation and subsequently reduces ovarian cancer cell growth, invasion and metastasis. Int J Cancer 2004;109:336-347.

14 Mukherjee A, Rotwein P: Insulin-like growth factor-binding protein-5 inhibits osteoblast differentiation and skeletal growth by blocking insulin-like growth factor actions. Mol Endocrinol 2008;22:1238-1250.

15 Sittadjody S, Ilangovan R, Thangasamy T, Vignesh RC, Veni S, Bertoni AG, Srinivasan S, Subramanian C, Srinivasan N: Age-related changes in serum levels of insulin-like growth factor-ii and its binding proteins correlate with calcaneal bone mineral density among post-menopausal south-indian women. Clin Chim Acta 2012;414:281-288.

-16 Chen L, Jiang W, Huang J, He BC, Zuo GW, Zhang W, Luo Q, Shi Q, Zhang BQ, Wagner ER, Luo J, Tang M, Wietholt C, Luo X, Bi Y, Su Y, Liu B, Kim SH, He CJ, Hu Y, Shen J, Rastegar F, Huang E, Gao Y, Gao JL, Zhou JZ, Reid RR, Luu HH, Haydon RC, He TC, Deng ZL: Insulin-like growth factor 2 (igf-2) potentiates bmp-9induced osteogenic differentiation and bone formation. J Bone Miner Res 2010;25:2447-2459.

17 Shang J, Yao Y, Fan X, Shangguan L, Li J, Liu H, Zhou Y: Mir-29c-3p promotes senescence of human mesenchymal stem cells by targeting cnot6 through p53-p21 and p16-prb pathways. Biochim Biophys Acta 2016;1863:520-532.

18 Lin YM, Wu CC, Chang YC, Wu CH, Ho HL, Hu JW, Chang RC, Wang CT, Ouyang P: Target disruption of ribosomal protein pno40 accelerates aging and impairs osteogenic differentiation of mesenchymal stem cells. Biochem Biophys Res Commun 2016;469:903-910.

19 Severino V, Alessio N, Farina A, Sandomenico A, Cipollaro M, Peluso G, Galderisi U, Chambery A: Insulinlike growth factor binding proteins 4 and 7 released by senescent cells promote premature senescence in mesenchymal stem cells. Cell Death Dis 2013;4:e911.

20 Guang LG, Boskey AL, Zhu W: Age-related cxc chemokine receptor-4-deficiency impairs osteogenic differentiation potency of mouse bone marrow mesenchymal stromal stem cells. Int J Biochem Cell Biol 2013;45:1813-1820.

-21 Zhang H, Li L, Dong Q Wang Y, Feng Q Ou X, Zhou P, He T, Luo J: Activation of pka/creb signaling is involved in bmp9-induced osteogenic differentiation of mesenchymal stem cells. Cell Physiol Biochem 2015;37:548562.

22 Kim BS, Kang HJ, Park JY, Lee J: Fucoidan promotes osteoblast differentiation via jnk- and erk-dependent bmp2-smad 1/5/8 signaling in human mesenchymal stem cells. Exp Mol Med 2015;47:e128.

-23 Nohe A, Keating E, Knaus P, Petersen NO: Signal transduction of bone morphogenetic protein receptors. Cell Signal 2004;16:291-299.

-24 Auh QS, Park KR, Yun HM, Lim HC, Kim GH, Lee DS, Kim YC, Oh H, Kim EC: Sulfuretin promotes osteoblastic differentiation in primary cultured osteoblasts and in vivo bone healing. Oncotarget 2016

25 Wu X, Chim SM, Kuek V, Lim BS, Chow ST, Zhao J, Yang S, Rosen V, Tickner J, Xu J: Htra1 is upregulated during rankl-induced osteoclastogenesis, and negatively regulates osteoblast differentiation and bmp2induced smad1/5/8, erk and p38 phosphorylation. FEBS Lett 2014;588:143-150.

26 Eskildsen T, Taipaleenmaki H, Stenvang J, Abdallah BM, Ditzel N, Nossent AY, Bak M, Kauppinen S, Kassem M: Microrna-138 regulates osteogenic differentiation of human stromal (mesenchymal) stem cells in vivo. Proc Natl Acad Sci U S A 2011;108:6139-6144.

27 Liu Y, Wu J, Zhu Y, Han J: Therapeutic application of mesenchymal stem cells in bone and joint diseases. Clin Exp Med 2014;14:13-24.

-28 Tokalov SV, Gruner S, Schindler S, Wolf G, Baumann M, Abolmaali N: Age-related changes in the frequency of mesenchymal stem cells in the bone marrow of rats. Stem Cells Dev 2007;16:439-446. 


\section{Cellular Physiology Cell Physiol Biochem 2017;42:640-650

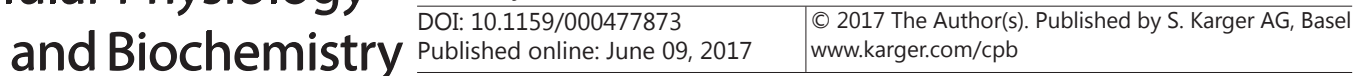 \\ Wu et al.: IGFBP4 Overexpression Inhibits Osteogenic Differentiation}

29 Minato A, Ise H, Goto M, Akaike T: Cardiac differentiation of embryonic stem cells by substrate immobilization of insulin-like growth factor binding protein 4 with elastin-like polypeptides. Biomaterials 2012;33:515-523.

-30 Xue Y, Yan Y, Gong H, Fang B, Zhou Y, Ding Z, Yin P, Zhang G, Ye Y, Yang C, Ge J, Zou Y: Insulin-like growth factor binding protein 4 enhances cardiomyocytes induction in murine-induced pluripotent stem cells. J Cell Biochem 2014;115:1495-1504.

- 31 Li H, Zuo S, Pasha Z, Yu B, He Z, Wang Y, Yang X, Ashraf M, Xu M: Gata-4 promotes myocardial transdifferentiation of mesenchymal stromal cells via up-regulating igfbp-4. Cytotherapy 2011;13:10571065.

32 Greenblatt MB, Shim JH, Glimcher LH: Mitogen-activated protein kinase pathways in osteoblasts. Annu Rev Cell Dev Biol 2013;29:63-79.

33 Choi H, Jeong BC, Kook MS, Koh JT: Betulinic acid synergically enhances bmp2-induced bone formation via stimulating smad 1/5/8 and p38 pathways. J Biomed Sci 2016;23:45.

-34 Wang J, Ding N, Li Y, Cheng H, Wang D, Yang Q, Deng Y, Yang Y, Ruan X, Xie F, Zhao H, Fang X: Insulin-like growth factor binding protein 5 (igfbp5) functions as a tumor suppressor in human melanoma cells. Oncotarget 2015;6:20636-20649.

-35 Zhong Y, Lu L, Zhou J, Li Y, Liu Y, Clemmons DR, Duan C: Igf binding protein 3 exerts its ligand-independent action by antagonizing bmp in zebrafish embryos. J Cell Sci 2011;124:1925-1935. 